\title{
Characterization of Toxoplasma gondii glyoxalase 1 and evaluation of inhibitory effects of curcumin on the enzyme and parasite cultures
}

Youn-Kyoung Goo', Junya Yamagishi' ${ }^{2}$, Akio Ueno ${ }^{3}$, Mohamad Alaa Terkawi ${ }^{3}$, Gabriel Oluga Aboge ${ }^{4}$, Dongmi Kwak', Yeonchul Hong ${ }^{1}$, Dong-ll Chung ${ }^{1}$, Makoto Igarashi ${ }^{3}$, Yoshifumi Nishikawa ${ }^{3}$ and Xuenan Xuan ${ }^{3^{*}}$

\begin{abstract}
Background: The glyoxalase pathway, which includes two enzymes, glyoxalase 1 and 2 (Glo1 and Glo2), is a ubiquitous cellular system responsible for the removal of cytotoxic methylglyoxal produced during glycolysis. Protozoan parasites, including Toxoplasma gondii (T. gondii) tachyzoites, produce methylglyoxal because of increased glycolytic fluxes. A Glo1 inhibitor such as curcumin could be considered a drug candidate for anti-protozoan, anti-inflammatory, and anti-cancer therapy.
\end{abstract}

Methods: The T. gondii Glo1 gene ( $\mathrm{TgGlo} 1$ ) was cloned and the recombinant protein was produced. Enzyme kinetics of TgGlo1 and five mutants were evaluated by adding methylglyoxal and glutathione to a reaction mixture. Finally, the inhibitory effects of various concentrations of curcumin on recombinant TgGlo1 were evaluated using in vitro cultures of $T$. gondii.

Results: Active recombinant TgGlo1 was successfully produced and the active sites (E166 and E251) of TgGlo1 were verified by point mutagenesis. Curcumin at the tested doses inhibited the enzymatic activity of recombinant TgGlo1 as well as the parasitic propagation of in vitro-cultured T. gondii. The $K_{i}$ and $I C_{50}$ were $12.9 \pm 0.5 \mu \mathrm{M}$ and $38.3 \pm 0.9 \mu \mathrm{M}$, respectively.

Conclusion: The inhibitory effect of curcumin on the enzymatic activity of TgGlo1 and parasitic propagation of T. gondii could be explored in the potential development of a potent drug for the treatment of toxoplasmosis. However, considering the fact that curcumin is known to have many effects on other molecules in the micromolar range, further elucidation of curcumin's direct inhibition of the glyoxalase system of T. gondii will be needed.

Keywords: Toxoplasma gondii, Glyoxalase pathway, Curcumin

\section{Background}

Toxoplasma gondii is a major pathogen of a broad range of warm-blooded animals, including humans, livestock, and domestic pets $[1,2]$. The parasite is of clinical importance in causing devastating disease in the developing fetus, as well as opportunistic infections in immunocompromised and transplant patients [3]. In human hosts, the parasites differentiate into tachyzoites and bradyzoites,

\footnotetext{
* Correspondence: gen@obihiro.ac.jp

${ }^{3}$ National Research Center for Protozoan Diseases, Obihiro University of Agriculture and Veterinary Medicine, Obihiro, Hokkaido 080-8555, Japan Full list of author information is available at the end of the article
}

which display distinct physiological features. The dormant bradyzoites show an extremely reduced growth rate, close to complete arrest of the cell cycle, while tachyzoites rapidly duplicate in the acute phase of infection. The tachyzoite stage constitutes an effective pathway for nutrient acquisition and energy metabolism for the parasite.

The glyoxalase pathway is the ubiquitous cellular system responsible for the removal of methylglyoxal, a cytotoxic reactive carbonyl compound produced in many organisms as a consequence of central metabolism [4-6]. During glycolysis, methylglyoxal is produced by imperfect degradation of glucose because of 
the spontaneous elimination of phosphate from dihydroxyacetone-phosphate or glyceraldehyde-3-phosphate [7] Plasmodium species, which are apicomplexan parasites closely related to $T$. gondii, also show increased methylglyoxal production [8, 9]. Therefore, the increased glycolytic fluxes in $T$. gondii tachyzoites would suggest that these parasites require an efficient detoxification system for removal of harmful methylglyoxal. While there are several reports of studies focusing on the glyoxalase system of Plasmodium species as a potential drug target, no such study has been reported in T. gondii to date [10].

Curcumin is a polyphenol derivative isolated from the plant Curcuma longa. By interacting with more than 30 proteins, curcumin exhibits anti-inflammatory, antioxidant, anti-viral, and anti-infectious activity in extensive studies conducted over the past several decades [11]. Curcumin was recently reported to be a nonglutathione inhibitor of small homodimeric mammalian glyoxalase 1 (Glo1) [12]. In addition, curcumin showed in vitro and in vivo anti-trypanosomal effects in Trypanosoma evansi as well as anti-malarial effects in Plasmodium falciparum cultures and in Plasmodium berghei in vivo [13-15]. In these studies, the effects of curcumin appeared to target the glyoxalase system, as suggested by its inhibition of the enzymatic activities of Glo1 of the Trypanosoma and Plasmodium species.

In the present study, we identified a functional Glo1 of T. gondii (TgGlo1) and evaluated active sites of TgGlo1 by point mutagenesis and enzyme kinetic analysis. In addition, inhibitory effects of curcumin against the enzymatic activity of recombinant TgGlo1 and parasite growth in $T$. gondii cultures were analyzed.

\section{Methods}

\section{Parasites and cell cultures}

The T. gondii $\mathrm{RH}$ and green fluorescent proteinexpressing RH (GFP-RH) strains used in this study were maintained in Vero cells and human foreskin fibroblasts (HFFs) at $37{ }^{\circ} \mathrm{C}$ and $5 \% \mathrm{CO}_{2}$ in Dulbecco's modified eagle medium (DMEM, Sigma-Aldrich, St. Louis, MO USA) supplemented with $10 \%$ heat-inactivated fetal bovine serum (FBS).

\section{Cloning of the TgGlo1 gene}

TgGlo1 was amplified from T. gondii cDNA using the primers listed in Table 1. The specific primers were designed according to the TgGlo1 sequence in ToxoDB (accession number, TGGT1_248400). The amplified polymerase chain reaction (PCR) products were extracted using the QIAquick gel extraction kit (QIAGEN, Hilden, Germany) and then digested with EcoRI and HindIII. The digested DNA was inserted between the EcoRI and HindIII sites of the expression vector pRSETb
Table 1 Primers used for amplification of TgGlo1 gene and generation of point mutations of TgGlo1

\begin{tabular}{|c|c|c|}
\hline Gene & Primer & Sequence \\
\hline \multirow[t]{2}{*}{ TgGlo1 } & Forward & 5'-GGGGAATTCATGTCCAGAAGTTTGCCC-3' \\
\hline & Reverse & 5'-GGGAAGCTTTTACTTCTTTGAAAGGAACG-3' \\
\hline \multirow[t]{2}{*}{ E92Q } & Forward & 5'-GGGACGTGCTTGCAACTCACTCACAATC-3' \\
\hline & Reverse & 5'-GATTGTGAGTGAGTTGCAAGCACGTCCC-3' \\
\hline \multirow[t]{2}{*}{ E166Q } & Forward & 5'-GGATACTGGATACAATTGGTTTCTCGG-3' \\
\hline & Reverse & 5'-CCGAGAAACCAATTGTATCCAGTATCC-3' \\
\hline \multirow[t]{2}{*}{ R188E } & Forward & 5'-CAGACGATGATTGAAATCAAAGATCCG-3' \\
\hline & Reverse & 5'-CGGATCTTTGATCCGAATCATCGTCTG-3' \\
\hline \multirow[t]{2}{*}{ E251Q } & Forward & 5'-CAGCCTGTATTACAACTCACACACAATCAC-3' \\
\hline & Reverse & 5'-GTGATTGTGTGTGAGTTGTAATACAGGCTG-3' \\
\hline \multirow[t]{2}{*}{ E324Q } & Forward & 5'-GGATATAGCATCCAACTGATTCAG-3' \\
\hline & Reverse & 5'-CTGAATCAGTTGGATGCTATATCC-3' \\
\hline
\end{tabular}

(Invitrogen, Carlsbad, CA, USA). Thereafter, accurate gene insertion was confirmed by digestion with restriction enzymes and sequencing.

\section{Generation of TgGlo1 point mutants}

Mutations of the TgGlo1 gene (E92Q, E166Q, R188E, E251Q, E324Q, E92Q/E324Q, and E166Q/E251Q) were introduced by PCR using Ex Taq polymerase (Takara, Shiga, Japan) with mutated primers (Table 1) and the TgGlo1 gene as a template. The mutant genes were subcloned into the pGEM T-easy vector (Promega, Madison, WI, USA) and subsequently confirmed by sequencing both strands. The mutated genes were subcloned into the pRSETb vector (Invitrogen) for recombinant protein expression.

\section{Expression and protein purification of wild-type and mutants of TgGlo1}

The protein expression was performed as previously described by Deponte et al. [16] with modifications. The pRSETb constructs of the wild-type and mutant TgGlo1 genes were expressed in E. coli strain BL21(DE3)pLysS (Invitrogen). Transformation of the respective plasmid to competent cells was performed before each expression, and the bacteria were precultured overnight at $37^{\circ} \mathrm{C}$. The preculture was added to fresh medium $(10 \mathrm{~g} / \mathrm{L}$ yeast extract, $5 \mathrm{~g} / \mathrm{L}$ peptone, $10 \mathrm{~g} / \mathrm{L} \mathrm{NaCl}, 2 \mathrm{~g} / \mathrm{L} \mathrm{MgSO}_{4} \cdot 7 \mathrm{H}_{2} \mathrm{O}$, $10 \mathrm{mM}$ 3-( $N$-morpholino) propanesulfonic acid (MOPS)/ $\mathrm{NaOH}$ at $\mathrm{pH} 7.5$, and $100 \mathrm{mg} / \mathrm{L}$ ampicillin) and grown at $37{ }^{\circ} \mathrm{C}$. After induction by $0.1 \mathrm{mM}$ isopropyl $\beta$-D-1-thiogalactopyranoside and overnight culturing, 31 of culture cells were harvested by centrifugation $(10 \mathrm{~min}$, $12,000 \times g$ ) and resuspended in $20 \mathrm{~mL}$ of buffer containing $10 \mathrm{mM}$ MOPS/NaOH (pH 7.8), lysozyme, DNase I, and $50 \mu \mathrm{M}$ phenylmethanesulfonyl fluoride. Then, the resuspended cells were sonicated at $4{ }^{\circ} \mathrm{C}$ and 
centrifuged for $30 \mathrm{~min}$ at $12,000 \times g$. The supernatant was applied to an $S$-hexylglutathione-agarose column (Sigma-Aldrich), which was equilibrated with buffer containing $10 \mathrm{mM}$ MOPS/NaOH (pH 7.8). After washing with eight column volumes of $10 \mathrm{mM}$ MOPS/ $\mathrm{NaOH}$ and $200 \mathrm{mM} \mathrm{NaCl}(\mathrm{pH} 7.8)$, the recombinant enzyme was eluted by three column volumes of $5 \mathrm{mM}$ $S$-hexylglutathione ( $\mathrm{pH}$ 7.8). The eluent was loaded onto a nickel-nitrilotriacetic acid column (Qiagen) and eluted with buffer containing $125 \mathrm{mM}$ imidazole (pH 8.0). The purity of rTgGlo1 was confirmed by SDSPAGE (Additional file 1: Figure S1).

\section{Production of antiserum against TgGlo1}

The antiserum specific to TgGlo1 was produced by immunizing five 6-week-old female ICR mice (CLEA Japan, Inc., Tokyo, Japan). The purified rTgGlo1 $(200 \mu \mathrm{g})$ were emulsified with an equal volume of complete Freund's adjuvant (Difco Laboratories, Detroit, MI, USA), and then intraperitoneally injected. Two additional boosters with $100 \mu \mathrm{g}$ of each antigen with incomplete Freund's adjuvant (Difco) were intraperitoneally administered at 2 -week intervals. The mice were bled 2 weeks after the last booster, and serum samples were stored at $-30{ }^{\circ} \mathrm{C}$. The specificity of anti-rTgGlo1 serum was evaluated by western blot as shown in Additional file 1: Figure S2.

\section{Steady-state kinetics}

The steady-state kinetics of the recombinant enzymes were monitored spectrophotometrically, using a thermostatted Hitach U-2001 visible spectrophotometer. Experiments to determine $K_{m}$ values were performed as described previously [16]. Stock solutions of $10 \mathrm{mM}$ glutathione (GSH; Sigma-Aldrich) in assay buffer containing $50 \mathrm{mM} \mathrm{MOPS/}$ $\mathrm{NaOH}(\mathrm{pH}$ 7.0) were freshly prepared before each experiment. For the desired concentration of hemithioacetal, concentrations of methylglyoxal and GSH in $1 \mathrm{~mL}$ of the assay mixture were calculated using the following equation.

$$
\begin{aligned}
\mathrm{Kd} & =3 \mathrm{mM} \\
& =([\text { methylglyoxal }][\mathrm{GSH}]) /[\text { hemithioacetal }]
\end{aligned}
$$

The calculated concentrations of free GSH after 5 min of preincubation and hemithioacetal were $0.1 \mathrm{mM}$ and 5-500 $\mu \mathrm{M}$, respectively. After the addition of enzyme, the formation of $S$-D-lactoylglutathione was measured spectrophotometrically at $240 \mathrm{~nm}\left(\varepsilon=3.37 \mathrm{mM}^{-1} \mathrm{~cm}^{-1}\right)$. The final assay concentration of the wild-type enzyme and mutants was 5-200 nM. The kinetic data of the initial reaction velocities were plotted according to the Lineweaver-Burk equation using the program SigmaPlot 10.0 (Systat Software Inc., San Jose, CA, USA).

\section{Enzymatic activity inhibition assays of curcumin}

The curcumin used for this assay was purchased from Sigma-Aldrich. Enzymatic activity of recombinant TgGlo1 was monitored at $30{ }^{\circ} \mathrm{C}$ using a thermostatted spectrophotometer. The TgGlo1 activity was determined at $240 \mathrm{~nm}$ by measuring the conversion of the hemithioacetal (the product of methylglyoxal and GSH) into $S$-D-lactoylglutathione $\left(\varepsilon=3.37 \mathrm{mM}^{-1} \mathrm{~cm}^{-1}\right)$. Stock solutions of $10 \mathrm{mM}$ curcumin in assay buffer $(50 \mathrm{mM}$ MOPS/NaOH pH 7.0) and less than $10 \mathrm{mM}$ curcumin in methanol were freshly prepared before each experiment. The curcumin was added after the first 4 min of the 5-min preincubation period and the reaction was initiated by adding $5 \mathrm{nM}$ of enzyme.

\section{Growth inhibition assay of curcumin-treated $T$. gondii}

Growth inhibition assay was performed following previously described methods with modifications [17-19]. Inhibition of parasite growth by curcumin was determined by counting the number of vacuoles containing $1,2,4$, 8 , or more than 16 tachyzoites. To investigate the effects of curcumin exposure on parasites, HFFs in 96-well plates were infected for $4 \mathrm{~h}$ and then treated with vehicle or decreasing concentrations $(25,15,10,5,2.5,1$, and $0.1 \mu \mathrm{M}$ ) of curcumin for $48 \mathrm{~h}$. Plates were harvested and analyzed by counting the number of vacuoles with different dividing stages of tachyzoites. In addition, in order to determine the $50 \%$ inhibitory concentration $\left(\mathrm{IC}_{50}\right)$ of curcumin against $T$. gondii cultures, HFFs infected with T. gondii expressing GFP were counted under a microscope. The $\mathrm{IC}_{50}$ represents the concentration of curcumin that was required for inhibition of $50 \%$ of $T$. gondii proliferation in the HFF.

\section{Ethics statement}

This study and all procedures were approved by the Animal Care and Use Committee of Obihiro University of Agriculture and Veterinary Medicine, Japan. The experimental animals were housed, fed, and given clean drinking water in accordance with the stipulated rules for the Care and Use of Research Animals Promulgated by Obihiro University of Agriculture and Veterinary Medicine, Japan.

\section{Results and discussion}

\section{Characterization of $T$. gondii glyoxalase 1}

TgGlo1 was amplified from cDNA of a $T$. gondii RH strain and contained a single open reading frame of 1008 nucleotides encoding a polypeptide of 336 amino acid residues. The sequence of TgGlo1 was identical to that previously reported (GenBank accession no. EPR63516). The purity of rTgGlo1 was confirmed by SDS-PAGE (Additional file 1: Figure S1). Two putative glyoxalase domains were identified at 20-167 and 181-325 
amino acids, by an open-sourced gene analysis program, SMART (http://smart.embl-heidelberg.de/). TgGlo1 shared homology with Glo1 of Neospora caninum (90 \%), Eimeria acervulina (64\%), Eimeria tenella (61 \%), and P. falciparum (50 \%). No signal peptide was predicted for TgGlo1 by SMART, indicating that it is likely a cytosolic protein. This notion was further supported by the immunofluorescence assay with antiserum against TgGlo1 using confocal microscopy, which revealed that TgGlo1 was located in the cytoplasm of $T$. gondii (Fig. 1), while preimmune serum did not react with $T$. gondii parasites (Additional file 1: Figure S3).

Evaluation of enzyme active sites of $T$. gondii glyoxalase 1 In order to evaluate the enzymatic activity of recombinant $\mathrm{TgGlo1}$, the calculated concentrations of methylglyoxal and GSH were mixed with rTgGlo1. As shown in Fig. 2, the plot for enzymatic activity of rTgGlo1 displayed a curved line and biphasic pattern. Therefore, regression plots were drawn in biphase (Fig. 2; 1/[S] = less than $40 \mu \mathrm{M}$ and $1 /[\mathrm{S}]=$ greater than $40 \mu \mathrm{M})$. The $\mathrm{K}_{\mathrm{m}}$ of phase $1\left(\mathrm{~K}_{\mathrm{m} 1}, 1 /[\mathrm{S}]=\right.$ less than $\left.40 \mu \mathrm{M}\right)$ and phase $2\left(\mathrm{~K}_{\mathrm{m} 2}\right.$, $1 /[\mathrm{S}]=$ greater than $40 \mu \mathrm{M})$ of rTgGlo1 was $10.0 \pm 0.6$ and $38.6 \pm 1.5 \mu \mathrm{M}$, respectively (Table 2 ). Subsequently, generating seven mutants at glutamate residues were tried by PCR, selected based on the active sites of $P$. falciparum Glo1 [16]. The conserved glutamate residues of Glo1 were previously reported to function in acid-base catalysis during substrate enediolate formation and reprotonation [20, 21]. Five mutants (E92Q, E166Q, R188E, E251Q, and E166Q/E251Q) were successfully generated; however, E324Q and E92Q/E324Q mutants were not prepared despite several trials. The five mutants were evaluated in enzyme activity assays and the enzyme kinetic plots of two mutants also displayed a biphasic pattern as shown in Table 2 and Fig. 2. The $\mathrm{K}_{\mathrm{m}}$ values of mutants were compared to that of wild-type TgGlo1, and E166Q and E251Q mutants showed higher $K_{m}$ values. Furthermore, $K_{m}$ of the paired mutants (E166Q/E251Q) showed the highest value. In addition, $\mathrm{K}_{\text {cat }}$ of the paired mutants was $2-10$-fold lower than that of wild-type enzyme. These results indicate that the glutamate residues located at positions 166 and 251 are crucial for enzymatic activity. In P. falciparum Glo1, the glutamate residue located on 345 amino acid was also reported to be the crucial functional active site. However, we did not succeed in producing the E324Q mutant of TgGlo1. Our results suggest that, the glutamate residues located on 166 and 251 amino acids of TgGlo1 are functional active sites of TgGlo1. The $P$. falciparum Glo1 was reported to possess two functionally paired glutamate residues located at positions 91/345 and 272/ 161 [16]. As shown in Table 2, the glutamate residues located on 166 and 251 amino acids of TgGlo1 would be functionally coupled. However, it is not clear if glutamate residues on 92 and 345 amino acids are functionally
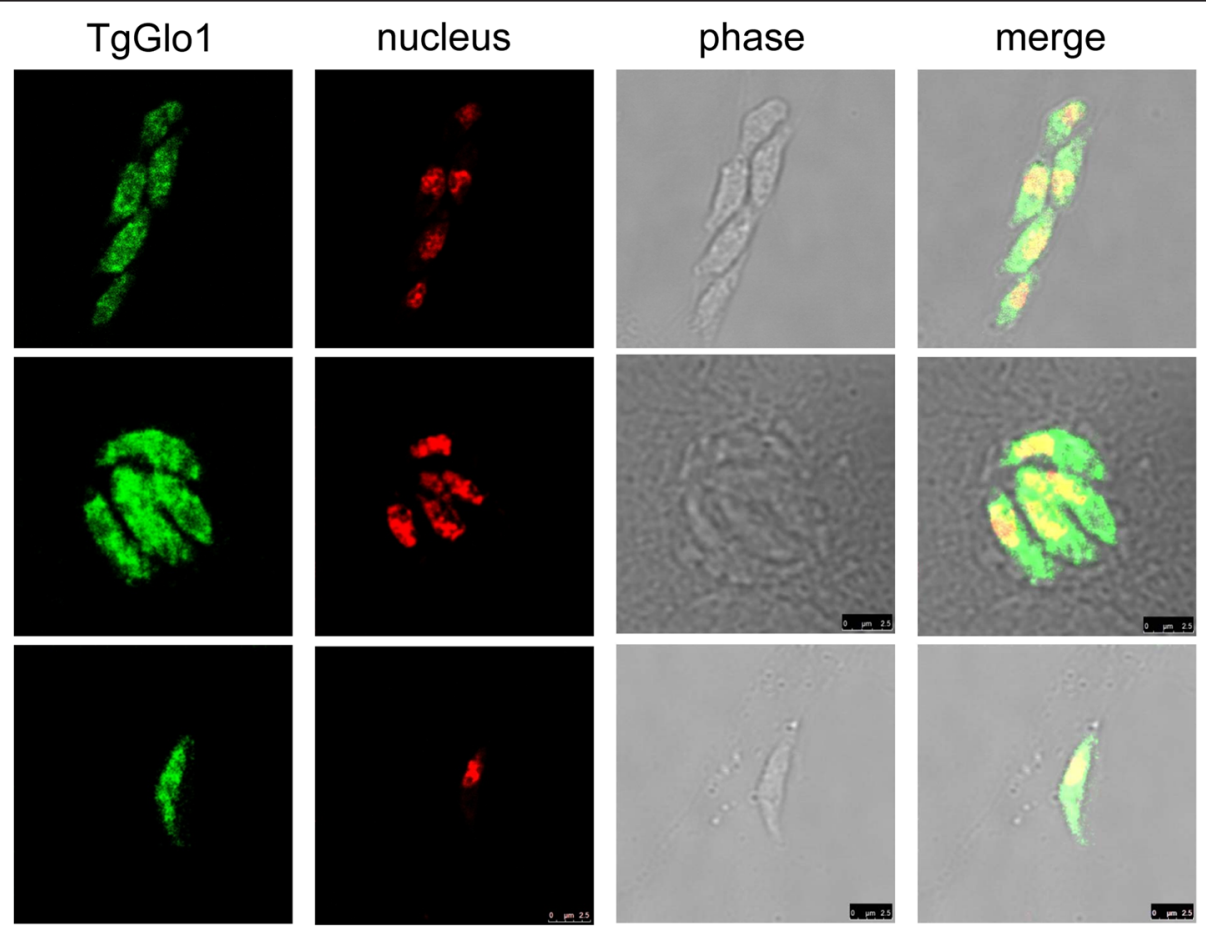

Fig. $1 \mathrm{Imm}$ unofluorescence microscopy analysis. The specific reaction of the rTgGlo1 and mouse anti-rTgGlo1 serum is green and the nuclei are red. Merged image of fluorescent green reactivity and red PI staining of nuclei with phase-contrast images of the parasites 

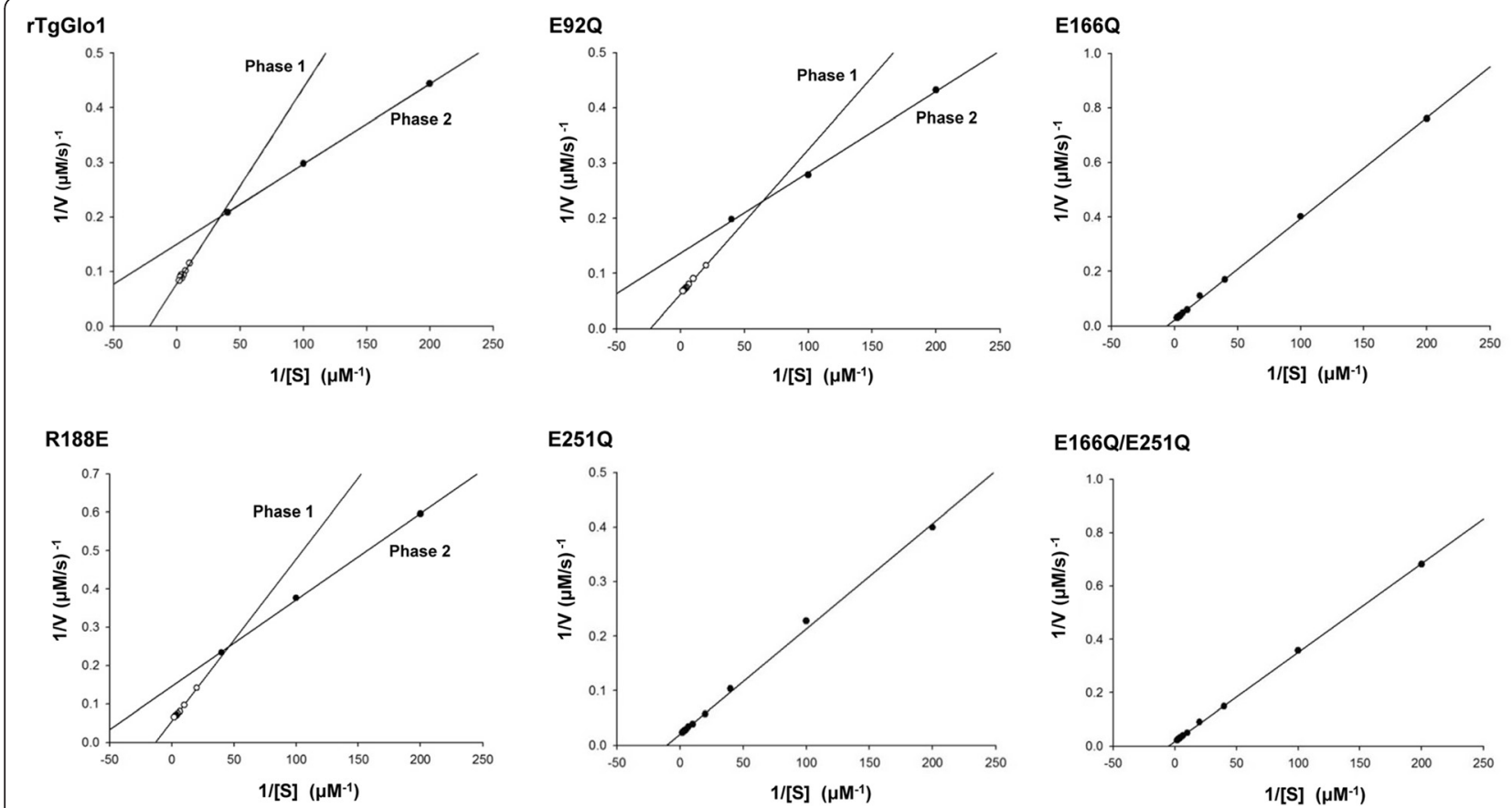

Fig. 2 Steady-state kinetics of wild-type rTgGlo1 and five mutants, E92Q, E166Q, R188E, E251Q, and E166Q/E251Q. Measurements are expressed as the mean of three independent experiments. The values for wild-type rTgGlo1, E92Q, and R188E were obtained from two phases (phase 1, 1/ $[\mathrm{S}]=$ less than $40 \mu \mathrm{M}$; phase $2,1 /[\mathrm{S}]=$ greater than $40 \mu \mathrm{M}$ )

paired, because of the lack of E345Q mutant production. To further evaluate the structural interaction of the residues, studies elucidating the chemical structure of TgGlo1 will be required.

\section{Inhibition of $T$. gondii glyoxalase 1 by curcumin}

Curcumin has been reported to have various pharmacological properties including anti-inflammatory, antiinfectious, and anti-carcinogenic [22]. A number of studies have proposed targets for the pharmacological effects including, transcription factors, enzymes, cytokines, and cell receptors. Glo1 has been considered one of the targets of curcumin in previous studies $[12,17]$. Curcumin acts by mimicking hemithioacetal, the enediolate reaction intermediate between methylglyoxal and GSH. Therefore, we investigated the inhibitory effects of curcumin on the enzymatic activity of rTgGlo1 by analyzing the steady-state kinetics at decreasing concentrations of substrate and curcumin. As shown in Fig. 3a,
Lineweaver-Burk plots indicate competitive inhibition with a constant maximum reaction velocity. The inhibitory effects were consistent in various timings of curcumin addition, from 1 to 4 min (data not shown). Curcumin has a similar structure to hemithioacetal, and it appears that curcumin directly interacts with the active site of TgGlo1. In addition, the $K_{i}$ value of curcumin against rTgGlo1 was $12.9 \pm 0.5 \mu \mathrm{M}$, and curcumin inhibition became insignificant at substrate concentrations greater than $125 \mu \mathrm{M}$ as shown in Additional file 1: Figure S4. Moreover, the enzymatic activity of rTgGlo1 was not completely inhibited by $50 \mu \mathrm{M}$ curcumin.

We also investigated the effects of curcumin in in vitro cultures of $T$. gondii. The number of vacuoles with more than 16 parasites was remarkably decreased at concentrations higher than $5 \mu \mathrm{M}$ (Fig. 3b). Specifically, a few vacuoles with many parasites (more than 8 ) were observed in the cultures treated with $10 \mu \mathrm{M}$ curcumin, compared to control cultures without curcumin (Fig. 3b).

Table 2 Enzyme kinetics of recombinant TgGlo1 proteins: a wild type and mutants produced by point-mutagenesis on active sites of TgGlo1

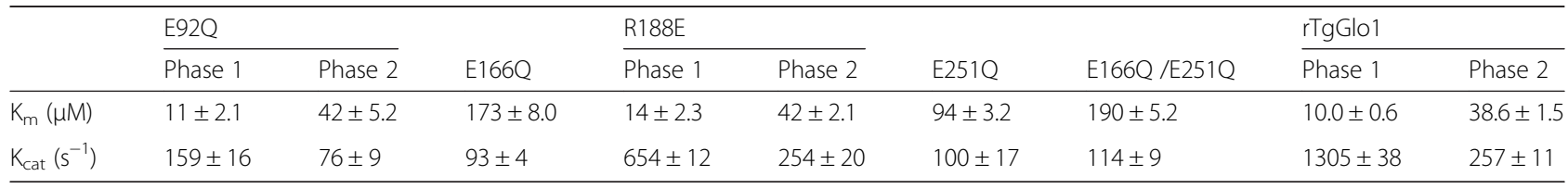

(Phase 1, 1/[S] = less than $40 \mu \mathrm{M}$; Phase 2, 1/[S] = more than $40 \mu \mathrm{M}$ ) 
A

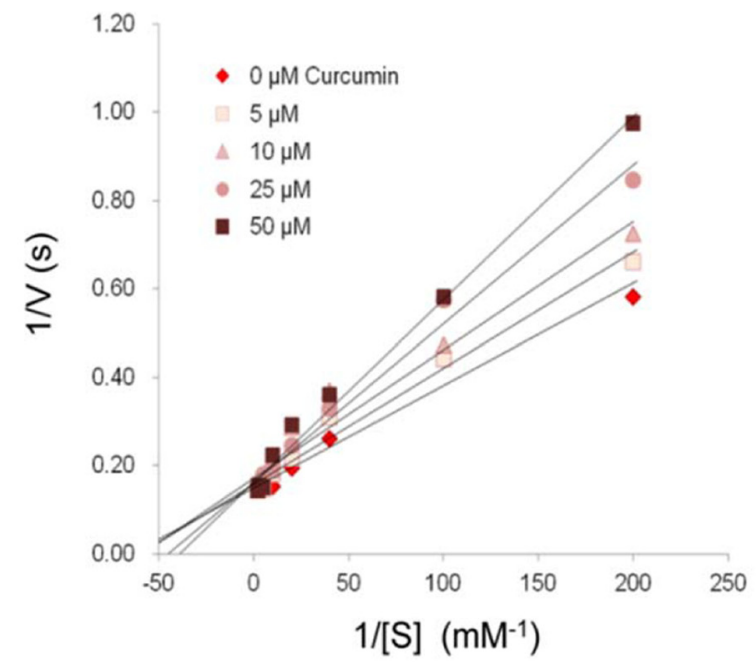

B

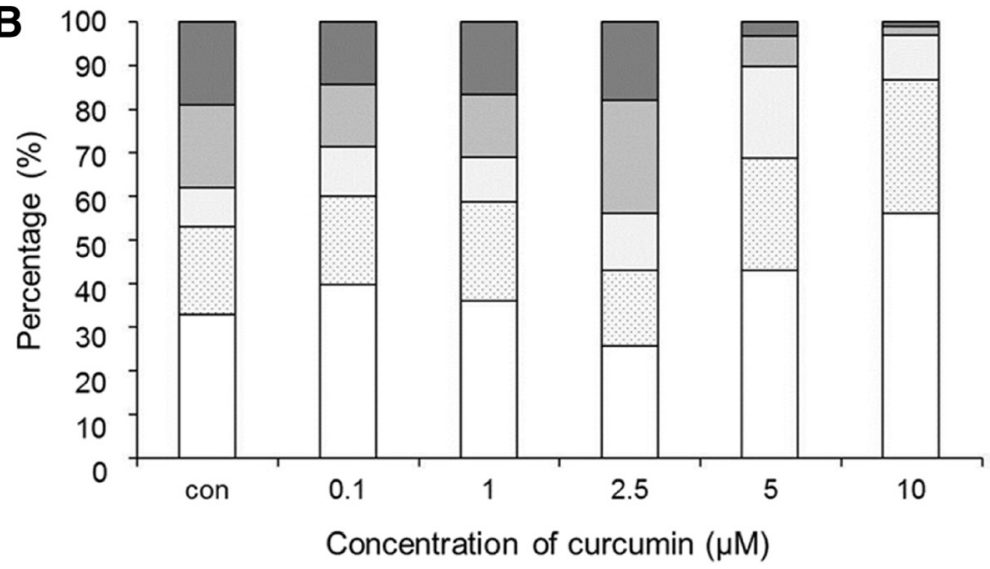

C

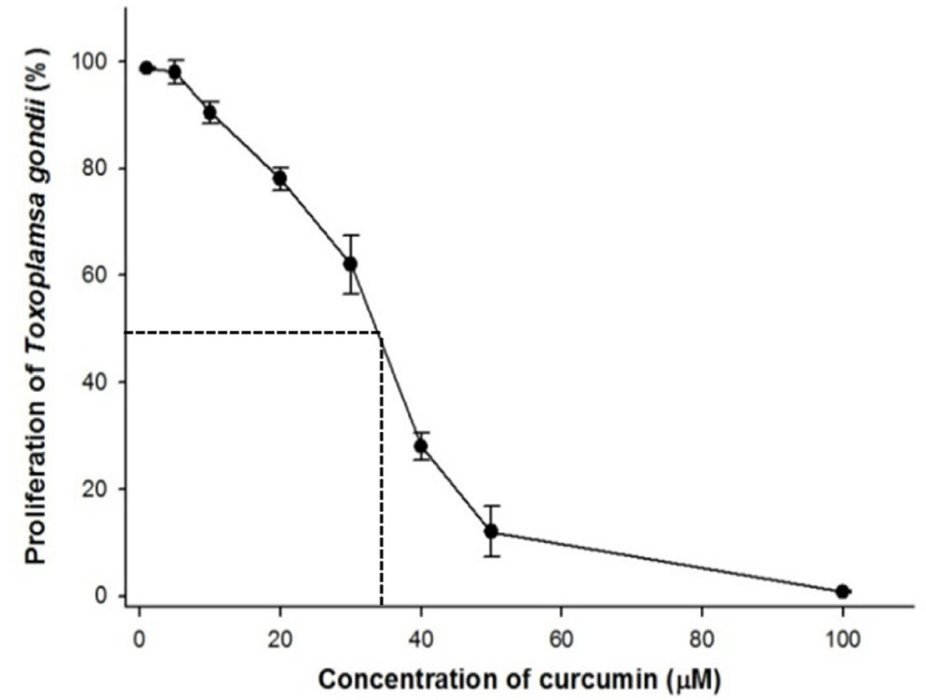

Fig. 3 Inhibition of TgGlo1 enzymatic activity and parasite growth of T. gondii by curcumin. a Inhibitory effects of curcumin on steady-kinetics of rTgGlo1. The purified rTgGlo1 was incubated with decreasing concentrations of curcumin and enzymatic activity was recorded. Enzymatic activity of rTgGlo1 decreased in a dose-dependent manner after treatment with curcumin. b Curcumin inhibition study in T. gondii cultures. T. gondii propagation was inhibited with concentrations of curcumin higher than $5 \mu \mathrm{M}$. c Curcumin inhibition study in dose-response experiments with T. gondii. Proliferation of T. gondii was decreased in a dose-dependent manner after treatment with curcumin 
When a broad range of curcumin concentrations (1$100 \mu \mathrm{M}$ ) was tested, the growth of $T$. gondii parasites was inhibited in a dose-dependent manner (Fig. $3 \mathrm{c}$ ). The $\mathrm{IC}_{50}$ of curcumin in T. gondii was $38.3 \pm 0.9 \mu \mathrm{M}$. These results suggest that curcumin may kill the $T$. gondii parasites by interfering with their differentiation. However, it is difficult to conclude that the growth inhibitory effect of curcumin on $T$. gondii was solely the result of targeting TgGlo1, because many effects of curcumin have been reported in the micromolar range [13]. To clarify this point, further evaluation of the direct inhibitory effects of curcumin on the glyoxalase system of $T$. gondii will be needed.

Rapidly propagating cells, including cancer cells and protozoan parasites, consume more glucose and have increased glycolytic flux compared to normal cells, resulting in intracellular levels of methylglyoxal that are toxic to the respective cells [23]. Methylglyoxal has been reported to modify the function of distinct proteins including heat shock protein 27, NF-kB, and glyceraldehyde 3-phosphate dehydrogenase in mammalian cells [24, 25]. Modulation of the proteins causes cell death by depletion of ATP, induction of apoptosis, and increases in levels of reactive oxygen species [26]. In order to remove the toxic methylglyoxal, the cells increase expression of Glo1. Therefore, inhibitors of Glo1 have potential as chemotherapeutic agents.

\section{Conclusion}

In the current study, a cytosolic TgGlo1 was identified and its active sites (E166 and E251) were verified by point mutagenesis. Curcumin inhibited the enzymatic activity of recombinant $\mathrm{TgGlo} 1$ and parasite propagation in cultures of $T$. gondii. However, considering the fact that curcumin is known to have many effects on other molecules in the micromolar range, further elucidation of curcumin's direct inhibition of the glyoxalase system of T. gondii will be needed.

\section{Additional file}

Additional file 1: Figure S1. SDS-PAGE of recombinant TgGlo1-His protein (12\% SDS-PAGE gel). Lane M, low molecular mass marker; lane A, recombinant TgGlo1-His protein; lane $B$, nickel-nitrilotriacetic acid resin after an elution of recombinant TgGlo1-His protein; lane C, Escherichia coli expressing recombinant TgGlo1-His after sonication. Figure S2. Western blot analysis of recombinant and native TgGlo1. Lane A, recombinant TgGlo1 reacted with anti-TgGlo1 mouse serum; lane B, Toxoplasma gondii lysate reacted with antiTgGlo1 mouse serum; lane C, T. gondii lysate reacted with pre-immunized mouse serum. Figure S3. Immunofluorescence staining and confocal microscopy for pre-immunized mouse serum. Pre-immunized mouse serum did not react with Toxoplasma gondii parasites. Merged image of fluorescent green reactivity and red PI staining of nuclei with phase-contrast images of the parasites showed only red-stained nuclei. Figure S4. The inhibitory effects of curcumin on enzymatic activity of rTgGlo1 with different concentrations of hemithioacetal $(5-250 \mu \mathrm{M})$. (DOC $551 \mathrm{~kb})$

\section{Competing interests}

The authors declare that they have no proprietary, commercial, or financial interests that could be construed to have inappropriately influenced this study.

\section{Authors' contributions}

YKG and XX designed the study and revised the manuscript. YKG, JY, AU MAT, and GOA performed the experiments. YKG, YN, and XX analyzed the data and drafted the manuscript. DMK, YH, DIC, and MI helped design the study and revise the manuscript. All authors read and approved the final manuscript.

\section{Acknowledgements}

This work was supported by a grant from the Global COE Program (J02) and a Grant-in-Aid for Scientific Research (A-22248035), both from the Ministry of Education, Culture, Sports, Science, and Technology, Japan. Y.K.G., M.A.T., and G.O.A. were supported by a research grant fellowship from the Japanese Society for the Promotion of Science (JSPS) for young scientists, Japan.

\section{Author details}

${ }^{1}$ Department of Parasitology and Tropical Medicine, Kyungpook National University School of Medicine, Daegu 700-422, Republic of Korea. ${ }^{2}$ Research Center for Zoonosis Control, Hokkaido University, Sapporo, Hokkaido 001-0020, Japan. ${ }^{3}$ National Research Center for Protozoan Diseases, Obihiro University of Agriculture and Veterinary Medicine, Obihiro, Hokkaido 080-8555, Japan. ${ }^{4}$ Department of Public Health, Pharmacology and Toxicology, University of Nairobi, P.O. BOX 2905300625 Kangemi, Nairobi, Kenya. ${ }^{5}$ Department of Veterinary Medicine, College of Veterinary Medicine and Stem Cell Research Therapeutic Institute, Kyungpook National University, Daegu 700-701, Republic of Korea.

Received: 16 March 2015 Accepted: 14 December 2015

Published online: 23 December 2015

\section{References}

1. Dubey JP. History of the discovery of the life cycle of Toxoplasma gondii. Int J Parasitol. 2009;39(8):877-82.

2. Gebremedhin EZ, Tadesse G. A meta-analysis of the prevalence of Toxoplasma gondii in animals and humans in Ethiopia. Parasit Vectors. 2015;8:291.

3. Luft BJ, Remington JS. Toxoplasmic encephalitis in AIDS. Clin Infect Dis. 1992;15(2):211-22

4. Hasim S, Hussin NA, Alomar F, Bidasee KR, Nickerson KW, Wilson MA. A glutathione-independent glyoxalase of the DJ-1 superfamily plays an important role in managing metabolically generated methylglyoxal in Candida albicans. J Biol Chem. 2014;289(3):1662-74.

5. Martins AM, Mendes P, Cordeiro C, Freire AP. In situ kinetic analysis of glyoxalase I and glyoxalase II in Saccharomyces cerevisiae. Eur J Biochem. 2001;268(14):3930-6.

6. Urscher M, Alisch R, Deponte $\mathrm{M}$. The glyoxalase system of malaria parasites-implications for cell biology and general glyoxalase research. Semin Cell Dev Biol. 2011:22(3):262-70.

7. Thornalley PJ. Protein and nucleotide damage by glyoxal and methylglyoxal in physiological systems-role in ageing and disease. Drug Metabol Drug Interact. 2008;23(1-2):125-50.

8. Sousa Silva M, Ferreira AE, Gomes R, Tomas AM, Ponces Freire A, Cordeiro C. The glyoxalase pathway in protozoan parasites. Int J Med Microbiol. 2012;302(4-5):225-9.

9. Kehr S, Sturm N, Rahlfs S, Przyborski JM, Becker K. Compartmentation of redox metabolism in malaria parasites. PLoS Pathog. 2010;6(12):e1001242.

10. Urscher M, More SS, Alisch R, Vince R, Deponte M. Tight-binding inhibitors efficiently inactivate both reaction centers of monomeric Plasmodium falciparum glyoxalase 1. FEBS J. 2012:279(14):2568-78.

11. Aggarwal BB, Sung B. Pharmacological basis for the role of curcumin in chronic diseases: an age-old spice with modern targets. Trends Pharmacol Sci. 2009;30(2):85-94.

12. Santel T, Pflug G, Hemdan NY, Schafer A, Hollenbach M, Buchold M, et al. Curcumin inhibits glyoxalase 1: a possible link to its anti-inflammatory and anti-tumor activity. PLoS One. 2008;3(10):e3508.

13. Cui L, Miao J. Cytotoxic effect of curcumin on malaria parasite Plasmodium falciparum: inhibition of histone acetylation and generation of reactive oxygen species. Antimicrob Agents Chemother. 2007;51(2):488-94.

14. Gressler LT, Oliveira CB, Coradini K, Rosa LD, Grando TH, Baldissera MD, et al. Trypanocidal activity of free and nanoencapsulated curcumin on Trypanosoma evansi. Parasitology. 2015;142(3):439-48. 
15. Reddy RC, Vatsala PG, Keshamouni VG, Padmanaban G, Rangarajan PN Curcumin for malaria therapy. Biochem Biophys Res Commun. 2005;326(2):472-4.

16. Deponte M, Sturm N, Mittler S, Harner M, Mack H, Becker K. Allosteric coupling of two different functional active sites in monomeric Plasmodium falciparum glyoxalase I. J Biol Chem. 2007;282(39):28419-30.

17. Urscher M, Przyborski JM, Imoto M, Deponte M. Distinct subcellular localization in the cytosol and apicoplast, unexpected dimerization and inhibition of Plasmodium falciparum glyoxalases. Mol Microbiol. 2010;76(1):92-103.

18. Saleh A, Friesen J, Baumeister S, Gross U, Bohne W. Growth inhibition of Toxoplasma gondii and Plasmodium falciparum by nanomolar concentrations of 1-hydroxy-2-dodecyl-4(1H)quinolone, a high-affinity inhibitor of alternative (type II) NADH dehydrogenases. Antimicrob Agents Chemother. 2007;51(4):1217-22.

19. Dzitko K, Paneth A, Plech T, Pawelczyk J, Weglinska L, Paneth P. Triazole-based compound as a candidate to develop novel medicines to treat toxoplasmosis. Antimicrob Agents Chemother. 2014;58(12):7583-5.

20. Himo F, Siegbahn PE. Catalytic mechanism of glyoxalase I: a theoretical study. J Am Chem Soc. 2001;123(42):10280-9.

21. Richter U, Krauss M. Active site structure and mechanism of human glyoxalase I-an ab initio theoretical study. J Am Chem Soc. 2001;123(29):6973-82.

22. Shishodia S, Sethi G, Aggarwal BB. Curcumin: getting back to the roots. Ann N Y Acad Sci. 2005;1056:206-17.

23. de Arriba SG, Stuchbury G, Yarin J, Burnell J, Loske C, Munch G. Methylglyoxal impairs glucose metabolism and leads to energy depletion in neuronal cells-protection by carbonyl scavengers. Neurobiol Aging. 2007;28(7):1044-50.

24. Lee CC, Lee BH, Wu SC. Actinidia callosa peel (kiwi fruit) ethanol extracts protected neural cells apoptosis induced by methylglyoxal through Nrf2 activation. Pharm Biol. 2014;52(5):628-36.

25. Sakamoto H, Mashima T, Yamamoto K, Tsuruo T. Modulation of heat-shock protein 27 (Hsp27) anti-apoptotic activity by methylglyoxal modification. J Biol Chem. 2002;277(48):45770-5.

26. Morin D, Barthelemy S, Zini R, Labidalle S, Tillement JP. Curcumin induces the mitochondrial permeability transition pore mediated by membrane protein thiol oxidation. FEBS Lett. 2001:495(1-2):131-6.

\section{Submit your next manuscript to BioMed Central and we will help you at every step:}

- We accept pre-submission inquiries

- Our selector tool helps you to find the most relevant journal

- We provide round the clock customer support

- Convenient online submission

- Thorough peer review

- Inclusion in PubMed and all major indexing services

- Maximum visibility for your research

Submit your manuscript at www.biomedcentral.com/submit

) Biomed Central 